\title{
-NOTES-
}

\section{ON COMPRESSIBLE FLOW ABOUT BODIES OF REVOLUTION*}

By W. R. SEARS** (Northrop Aircraft, Inc.)

The linear-perturbation theory of compressible fluid flow originated by Glauert and Prandtl has recently been presented in a revised and clarified form by Goldstein and Young. ${ }^{1}$ These authors show three alternative procedures by which the compressible flow in an $x, y, z$-space can be deduced from a corresponding incompressible flow.

The linearized differential equation satisfied by the velocity potential $\phi$ is

$$
\beta^{2} \frac{\partial^{2} \phi}{\partial x^{2}}+\frac{\partial^{2} \phi}{\partial y^{2}}+\frac{\partial^{2} \phi}{\partial z^{2}}=0
$$

where $\beta^{2}$ denotes $1-U^{2} / a^{2}, U$ and $a$ being the stream velocity and the velocity of sound, respectively, in the undisturbed parallel flow. If the solution of (1) for the case $\beta=1$ (incompressible) is $\phi=U x+f(x, y, z)$, corresponding solutions for $\beta<1$ are given by the following alternative forms:

$$
\begin{aligned}
\phi & =U x+\frac{1}{\beta} f(x, \beta y, \beta z) \\
\phi & =U x+f(x, \beta y, \beta z) \\
\phi & =U x+f(x / \beta, y, z) .
\end{aligned}
$$

Each of these variants represents a somewhat different compressible flow, but all three are related to the given incompressible flow. The results determined by the theory are consistent, of course, as far as the linear theory is applicable, and the procedure used in any given problem is the one that provides the greatest ease of calculation. For example, in I the geometry of a slender body remains unaltered as $\beta$ varies; in II the body is distorted but the pressures on its surface are unchanged; and so forth.

Method II is the one used by Tsien and Lees in a recent paper, ${ }^{2}$ while both I and II are presented by Liepmann and Puckett in a new textbook. ${ }^{3}$ Sauer ${ }^{4}$ writes, in effect,

$$
\phi=U x+\lambda f(x, \beta y, \beta z)
$$

and selects the value of $\lambda$ most convenient for any given problem; this includes both I and II. Finally, B. Göthert, ${ }^{5}$ rejecting II because of a fancied discrepancy (actually

* Received Jan. 14, 1946.

** Now at Cornell University.

${ }^{1}$ S. Goldstein and A. D. Young, The linear perturbation theory of compressible flow with applications to wind-tunnel interference, British Aero. Res. Com. Reports and Memoranda No. 1909 (1943).

${ }^{2}$ H. S. Tsien and L. Lees, The Glauert-Prandtl approximation for subsonic flow of a compressible fluid, J. Aero. Sci. 12, 173-187, 202 (1945).

${ }^{3} \mathrm{H}$. W. Liepmann and A. Puckett, Introduction to the aerodynamics of compressible fluids, John Wiley and Sons, New York, 1946.

4 R. Sauer, Theoretische Einführung in die Gasdynamik, Springer, Berlin, 1943. Reprinted by Edwards Bros., Inc., Ann Arbor, 1945.

- B. Göthert, Ebene und räumliche Strömung bei hohen Unterschallgeschwindigkeiten, Lilienthal Gesellschaft f. Luftfahrtforschung, Bericht 127, 97-101 (1940). 
caused by an error in his application of the method), introduces still another variant by writing

V)

$$
\phi=\beta^{2} U x+f(x, \beta y, \beta z) .
$$

We are particularly concerned here with the application of these procedures to the flow about bodies of revolution. There is some confusion on this subject: Goldstein and Young, ${ }^{1}$ using $I$, find that the superstream velocities at a streamline body in the absence of trailing vortices are $1 / \beta$ times those at the same location in incompressible flow, while both Sauer ${ }^{4}$ and Göther $t^{5}$ conclude that these velocities are unaffected by compressibility, at least for slender bodies. ${ }^{6}$

This confusion is partly due to the fact that, while the procedures are equivalent and must yield consistent results in the linear approximation, they may produce different results when they are applied outside this range. For example, let the maximum velocity at the surface of a slender body in incompressible flow be denoted by $U \cdot[1+F(n)]$ where $n$ is the ratio of maximum diameter to length, so that $F(n)$ is a given function for any family of bodies. The several variants of the theory then yield the following results for the maximum surface velocity (divided by the stream velocity) in the compressible flow:

$$
\frac{1}{\beta} F(n)
$$$$
F(n / \beta)
$$$$
\frac{1}{\beta} F(n)
$$

$$
\lambda F(n / \lambda \beta)
$$$$
\frac{1}{\beta^{2}} F(\beta n) \text {. }
$$

Obviously these results are all the same if $F(n)$ is proportional to $n$ or can be approximated successfully in that form. But for a typical family of bodies, the ellipsoids of revolution, $F(n)$ actually has the form ${ }^{7}$

$$
F(n)=\frac{n^{2} \log p-2 n^{2} \sqrt{1-n^{2}}}{2 \sqrt{1-n^{2}}-n^{2} \log p} \text { where } p=\frac{1+\sqrt{1-n^{2}}}{1-\sqrt{1-n^{2}}}
$$

or, neglecting terms of order $n^{2}$,

$$
F(n)=-n^{2} \log n .
$$

The absence of a linear term in this expression is what leads Göthert to the conclusion that there is no correction for compressibility. Sauer's similar conclusion apparently results somewhat analogously from the fact that he considers only the

"Göthert admits a correction "for greater thickness ratio" and proceeds to calculate it by means of Method V above.

7 This is obtained from H. Lamb, Hydrodynamics, Cambridge, 1932, §105. Note that the ratio diameter/length, $n$, is equal to $\sqrt{1-\zeta^{-2}}$ in Lamb's notation. 
limiting case $n \mathrm{M} 0$. Actually, in (2a) we have retained the leading term while neglecting $O\left(n^{2}\right)$, which is consistent with the linear-perturbation theory.

$F(n)$ according to (2) and (2a) is plotted in Figure 1. It is clear that Göthert's and Sauer's conclusion cannot be correct in the range of practical interest $(1 / 10<n<1 / 3$ and $0.6 \leqq \beta<1$, say), since all of the various procedures listed above result in appreciable corrections to the velocity ratio. It seems more reasonable to conclude merely that the linear-perturbation theory cannot distinguish between the various'results. In this situation the formula of Method I might well be adopted by reason of its simplicity.

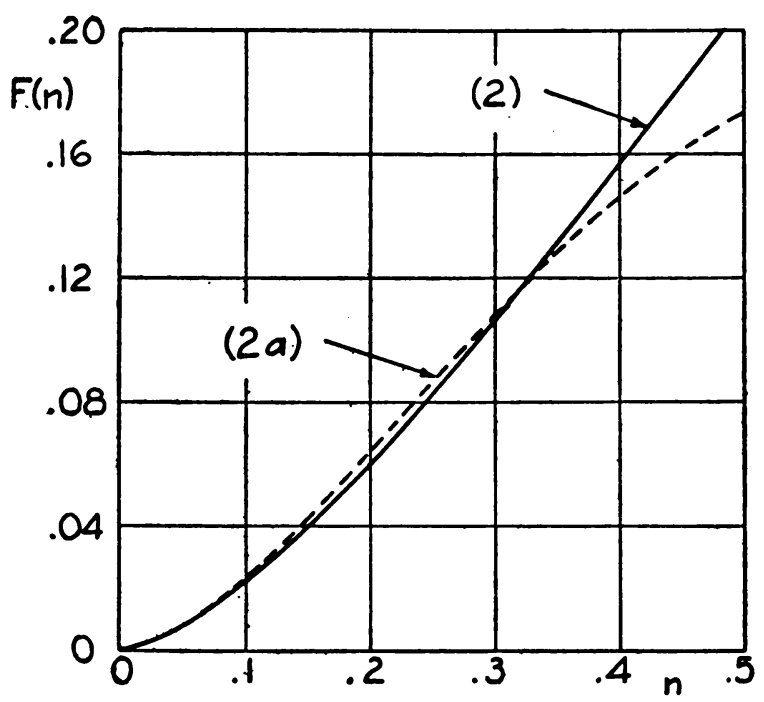

FIG. 1. The superstream velocity ratio for ellipsoids of revolution in incompressible flow.

\section{ON THE NUMERICAL TREATMENT OF FORCED OSCILLATIONS*}

\section{By ALVIN C. SUGAR** (Northrop Aircraft)}

1. Introduction. The differential equation, with typical initial conditions, of an harmonic oscillator subject to the action of a general disturbing force $m a(t)$ is given by

$$
\ddot{x}+\omega^{2} x=a(t), \quad x(0)=0=\dot{x}(0) .
$$

This equation occurs in problems involving from one to infinitely many degrees of freedom. Its solution can be expressed as follows:

$$
x=\frac{D}{\omega}, \quad \text { where } \quad D=\int_{0}^{t} a(\tau) \sin \omega(t-\tau) d \tau
$$

is the so-called Duhamel integral. If in (1) we replace only $x$ by $D / \omega$ we obtain an expression for the acceleration of the body.

$$
\ddot{x}=a(t)-\omega D .
$$

In this note a simple expression which is an approximation of $D$ is found. This expression provides a convenient process for evaluating $x$ and related quantities. Using the resulting simplified form of the acceleration a quick and easy vector method of obtaining the maximum acceleration is explained. Rapid methods of finding the

* Received Oct. 1, 1945.

** Now at Brown University. 